\title{
ORGANIZAÇÕES DE APOIO NO AUXÍLIO À GOVERNANÇA EM CLUSTERS COMPETITIVOS
}

\author{
iD Cristina Espinheira Costa Pereira ${ }^{1}$ (D) Ana Cláudia Azevedo² ${ }^{\text {(D) }}$ Ernesto Michelangelo Giglio $^{3}$ (iD João Maurício \\ Gama Boaventura ${ }^{4}$
}

\author{
${ }^{1}$ Doutora em Administração pela FEA-USP.Professora, Centro Universitário FAM e da Universidade Ibirapuera. cristina_ecp@hotmail.com \\ ${ }^{2}$ Doutora em Administração pela FEA-USP. Professora adjunta, Universidade Federal de Viçosa - UFV. anaazevedo@ufv.br \\ ${ }^{3}$ Doutor em Administração pela FEA-USP. Professor, UNIP. ernesto.giglio@ gmail.com \\ ${ }^{4}$ Doutor em Administração pela FEA-USP. Professor titular, FEA-USP. jboaventura@ usp.br
}

\section{Resumo}

Objetivo: Analisar como as organizações de apoio atuam sobre a governança, especificamente nos fatores competitivos: estratégias de resultado orientadas para o cluster, e caráter evolucionário por introdução de tecnologias.

Metodologia/Abordagem: Realizou-se um estudo de caso múltiplo nos clusters de Ciências da Saúde de Ribeirão Preto - Brasil, e o Oxfordshire Health Science Cluster - Inglaterra.

Originalidade/Relevância: Este estudo lança luz sob o potencial das organizações de apoio no desenvolvimento de atividades que reforcem a governança dos clusters, sobretudo em relação ao direcionamento estratégico e ao apoio à inovação.

Principais Resultados: Os resultados evidenciam e reforçam a importância das organizações de apoio para a governança e, sua influência positiva na competitividade. Em termos práticos evidencia a importância que tais organizações têm para o desenvolvimento de estratégias que favoreçam a coletividade, além de promover e disseminar o desenvolvimento tecnológico na região.

Contribuições teóricas/metodológicas: $\mathrm{O}$ trabalho contribui teoricamente ao sustentar, com evidências, a importância das organizações de apoio para a governança do cluster; avançando a compreensão sobre a teoria das redes de relacionamento, quando se consideram os laços mais fracos, na competitividade dos clusters. O benefício metodológico e gerencial consiste na oferta de uma matriz de seis atividades que podem ser investigadas em pesquisas futuras e/ou utilizadas por gestores dos clusters, indicando que tais ações podem e devem incluir as organizações de apoio.

Palavras-chave: Clusters. Governança. Organizações de apoio Competitividade.

\section{SUPPORT ORGANIZATIONS IN ASSISTING GOVERNANCE IN COMPETITIVE CLUSTERS}

\section{Abstract}

Objective: To analyze how support organizations act on governance, specifically on competitive factors: cluster-oriented outcome strategies, and evolutionary character through the introduction of technologies.

Methodology / Approach: A multiple case study was carried out in the Health Sciences clusters of Ribeirão Preto - Brazil, and the Oxfordshire Health Science Cluster - England.

Originality / Relevance: This study sheds light on support organizations potential to develop activities that reinforce clusters' governance, especially concerning strategic direction and support for innovation.

Main Results: The results show and reinforce the importance of support organizations for governance and their positive influence on competitiveness In practical terms, it highlights the importance that such organizations have for the development of strategies that favor the community and promote and disseminate technological development in the region.

Theoretical/methodological contributions: The work contributes theoretically by supporting, with evidence, the importance of support organizations for the cluster's governance, advancing the understanding of relationship network theory when considering the weakest ties in the competitiveness of clusters. The methodological and managerial benefit consists of offering a matrix of six activities that can be investigated in future research and/or used by the clusters' managers, indicating that such actions can and should include the support organizations.

Keywords: Clusters. Governance. Support organizations. Competitiveness.

\section{ORGANIZACIONES DE APOYO PARA AYUDAR A LA GOBERNANZA EN CLÚSTERES COMPETITIVOS}

\section{Resumen}

Objetivo: Analizar cómo actúan las organizaciones de apoyo en la gobernanza, específicamente en los factores competitivos: estrategias de resultados orientadas a clústeres y carácter evolutivo a través de la introducción de tecnologías.

Metodología / Enfoque: Se realizó un estudio de caso múltiple en los clústeres de Ciencias de la Salud de Ribeirão Preto - Brasil, y el Clúster de Ciencias de la Salud de Oxfordshire - Inglaterra.

Originalidad / Relevancia: Este estudio arroja luz sobre el potencial de las organizaciones de apoyo para desarrollar actividades que refuercen la gobernanza de los clústeres, especialmente en relación con la dirección estratégica y el apoyo a la innovación.

Principales Resultados: Los resultados muestran y refuerzan la importancia de las organizaciones de apoyo a la gobernanza y su influencia positiva en la competitividad. En términos prácticos, destaca la importancia que tienen estas organizaciones para el desarrollo de estrategias que favorezcan a la comunidad, además de promover y difundir el desarrollo tecnológico en la región.

Aportes teóricos/metodológicos: El trabajo contribuye teóricamente al respaldar, con evidencia, la importancia de las organizaciones de apoyo a la gobernanza del clúster; avanzar en la comprensión de la teoría de redes de relaciones, al considerar los lazos más débiles, en la competitividad de los clusters. El beneficio metodológico y gerencial consiste en ofrecer una matriz de seis actividades que pueden ser investigadas en futuras investigaciones y / o utilizadas por los gerentes de los clusters, indicando que tales acciones pueden y deben incluir a las organizaciones de apoyo.

Palabras-clave: Clusters. Gobernancia. Organizaciones de apoyo. Competitividad.

\section{Cite as / Como citar}

American Psychological Association (APA)

Pereira, C. E. C., Azevedo, A. C., Giglio, E. M., \& Boaventura, J. M. G. (2021, Special Issue, March). Organizações de apoio no auxílio à governança em clusters competitivos. Iberoamerican Journal of Strategic Management (IJSM), 20, p. 1-25, e16134. https://doi.org/10.5585/riae.v20i1.16134

PEREIRA, C. E. C.; AZEVEDO, A. C.; GIGLIO, E. M.; BOAVENTURA, J. M. G. Organizações de apoio no auxílio à governança em clusters competitivos. Iberoamerican Journal of Strategic Management (IJSM), v. 20, Special Issue, p. 1-25, e16134. Mar. 2021. https://doi.org/10.5585/riae.v20i1.16134. 
Há algumas décadas a literatura está caminhando para o entendimento quanto à importância da presença das organizações de apoio em clusters de negócios (Morosini, 2004; Wang, Madhok \& Li, 2014; Gereffi \& Lee, 2016). Essa análise passa pelo tema da governança, ou seja, dos mecanismos que regulam e incentivam a ação coletiva. A governança é importante para a criação, transferência e difusão de conhecimento geradores de inovação, que habilitam empresas a aprimorar sua performance (Cassanego, Boaventura, Azevedo \& Telles, 2019). Esse conhecimento advém de várias fontes, incluindo as organizações de apoio (Crona \& Parker, 2012).

Esses mecanismos da governança moderam as relações intra e interfirmas, a estrutura de ligações entre os atores da rede e os modos de solução das tarefas, o que se liga diretamente aos resultados das redes (Maccio \& Cristofoli, 2017). Quando a rede é local, ou seja, com uma estrutura de ligações entre atores geograficamente próximos, a literatura enfatiza a importância da governança no incremento e qualidade das interações entre as diversas organizações participantes da tarefa (Humphrey \& Schmitz, 2002), incluindo as organizações de suporte, como universidades e centros de pesquisa.

Nesse esforço de integração local, em certos casos, as associações de empresários e/ou o governo local criam centros de serviços especializados; reforçam a infraestrutura e lançam iniciativas para apoiar o cluster (Hoppen, Rigoni, Klein \& Ritter, 2016). Nesse movimento surgem organizações que desempenham papel relevante na provisão de mão de obra qualificada, serviços de treinamento e consultoria, e coalizão de P\&D (Li, Zubielqui \& O’Connor, 2015). Embora sejam organizações de apoio, elas suportam e disseminam valores socioculturais e econômicos comuns, em associação com organizações economicamente fortes e inovadoras em um cluster (Lefebvre, 2016).

Organizações como universidades, centros de pesquisa, associações industriais e institutos tecnológicos são exemplos dessas organizações de apoio que promovem a cooperação econômica mútua e a partilha de conhecimentos tecnológicos entre os membros de um cluster (Morosini, 2004). Embora sua presença e importância seja comentada e ressaltada na literatura (Li et al., 2015; Molina-Morales \& Martínez-Cháfer, 2016), a pesquisa não avançou na discussão acerca do papel e das ações dessas organizações perante a governança dos clusters (Ingstrup \& Damgaard, 2013; Mueller \& Jungwirth, 2016), especialmente sua relação com a atualização tecnológica dos arranjos e com a competitividade do grupo (Bocquet, Brion \& Mothe, 2016).

Considerando a importância do tema e a raridade de trabalhos empíricos, o benefício teórico do trabalho é o avanço na compreensão do papel das organizações de apoio no desenvolvimento de ações que reforcem a governança e a competitividade dos clusters, fundado em pesquisa de campo. Neste contexto, a pergunta orientadora de pesquisa é analisar como as organizações de apoio atuam sobre a governança supra empresarial, especificamente nos fatores competitivos de estratégias de resultado orientadas para o cluster, e caráter evolucionário por introdução de tecnologias (Zaccarelli et al., 2008).

Como desdobramentos desse objetivo principal pode-se elencar: 
(i) a verificação da presença de organizações de apoio e análise de suas respectivas participações na governança, no desenvolvimento de estratégias conjuntas orientadas para o aumento da competitividade do cluster;

(ii) a verificação da presença de organizações que apoiam a inovação e análise das suas respectivas ações de intervenção na governança, que estimulem o caráter evolucionário das empresas aglomeradas;

(iii) organização e proposição de uma matriz de atividades chaves sobre a participação de organizações de apoio para impulsionar a competitividade dos clusters.

Para tanto, foi realizada uma pesquisa em dois clusters de ciências da saúde pertencentes a dois países distintos: o cluster de Ciências da Saúde em Ribeirão Preto, Brasil, e, o Health Science Cluster em Oxforfdshire na Inglaterra.

Este artigo está organizado conforme orienta Ferreira (2013). Assim, além desta introdução, no tópico seguinte, o referencial teórico apresenta uma síntese da literatura que fundamenta o estudo. $\mathrm{Na}$ sequência é apresentada uma descrição sistematizada dos métodos adotados para consecução da pesquisa. O tópico subsequente traz a discussão dos resultados, com a proposição de um compilado envolvendo as atividades chaves a serem desenvolvidas pelas organizações de apoio para impulsionar a competitividade dos clusters. Por fim, tem-se as considerações finais com uma explanação geral, as contribuições do estudo, limitações e sugestões para pesquisas futuras.

\section{Referencial teórico}

Neste item apresentam-se os argumentos teóricos que fundamentam a construção da proposição da pesquisa e guiam as interpretações dos dados. As expressões chave que guiam a apresentação são cluster, competitividade e governança.

\section{Clusters e competitividade}

A trajetória de pesquisa envolvendo os agrupamentos empresariais geograficamente concentrados teve início no final do século XIX iniciada por Alfred Marshall, e fortalecida dentre outros pesquisadores, por Giacomo Beccatini; que observaram os distritos industriais anglo-saxões e italianos, ressaltando as externalidades positivas derivadas da proximidade geográfica e da ação conjunta (Cassanego et al., 2019). Tais externalidades podem ser atribuídas, principalmente, ao compartilhamento de bases institucionais e sociais comuns, dentro de um contexto de produção especializada e geograficamente agrupada (Petry, Amal \& Floriani, 2018; Parrilli \& Sacchetti 2008).

O termo cluster utilizado em referência a esses arranjos tornou-se popularmente conhecido a partir da década de 1990, quando o economista Michael Porter descreveu a estreita relação entre estes aglomerados industriais e a competitividade de empresas e nações (Porter, 1990; Porter, 2008). 
Sumarizando as contribuições realizadas ao longo do desenvolvimento da literatura, clusters podem ser definidos como entidades socioeconômicas, caracterizadas por concentrações geográficas de empresas interconectadas, fornecedores especializados, prestadores de serviços e organizações (por exemplo, universidades, agências governamentais, associações comerciais) que trabalham em conjunto em atividades economicamente vinculadas (Porter, 1998; Morosini, 2004). Note-se, na definição, que as organizações de apoio aparecem no mesmo patamar de importância das organizações mais diretamente envolvidas na tarefa.

Em linhas gerais, um cluster pode ser considerado mais ou menos competitivo à medida que apresenta alguns fatores fundamentais da performance competitiva, ou fundamentos de competitividade (Porter, 1990, 1998; Zaccarelli et al., 2008: Kamath, Agrawal \& Chase, 2012; Pereira, Sarturi, Boaventura \& Polo, 2014). Tais fatores variam desde a concentração geográfica, requisito primeiro para formação do cluster, até a presença de mão de obra especializada e cultura local, consideradas elementos intrínsecos à formação e desenvolvimento orgânico do arranjo (Lübeck, Wittmann \& da Silva, 2012).

Todavia, há de se considerar também, os fundamentos de competitividade que prescindem de um alinhamento estratégico deliberado, ou seja, demandam governança para existir (Zaccarelli et al., 2008). Neste escopo, são exemplos de fundamentos de competitividade em clusters: (i) estratégias de resultado voltadas para o cluster e, (ii) caráter evolucionário por introdução de tecnologias (Porter, 1990; Zaccarelli et al., 2008; Visser \& Atzema, 2008; Thomaz et al., 2011; Hoppen et al., 2016).

Define-se o fundamento estratégias de resultado voltadas para cluster como a "presença efetiva e deliberada de orientação para ação e decisão das empresas participantes do agrupamento" (Zaccarelli et al. 2008, p. 81). Ainda segundo os autores, uma estratégia orientada para o cluster pode ser identificada por acontecimentos como: reuniões entre negócios, programação de eventos, presença de assessoria específica e ações voltadas para a competitividade do agrupamento. Tais eventos, em geral, são organizados e difundidos pelas organizações de apoio ou suporte presentes no cluster, o que sustenta e qualifica o presente artigo.

Sobre o segundo ponto, as demandas por mudanças tecnológicas, também estão relacionadas a ações de governança e autores (Zaccarelli et al. 2008; Zhang \& Li, 2010) afirmam que sua ocorrência é inviável apenas por auto-organização e são necessárias condições que estimulem o desenvolvimento e a introdução de tecnologias. Ainda segundo autores, o caráter evolucionário pode ser constatado pela avaliação da tecnologia atual do cluster comparado com a existente em clusters semelhantes ou com as tecnologias mais avançadas disponíveis. Sobre este segundo ponto é possível inferir, já que não está explícito, que organizações de apoio podem auxiliar, através de seus contatos com outras organizações de outros clusters.

Em linhas gerais, pode-se agrupar os recursos que influenciam a competitividade de clusters em três conjuntos: recursos locais, recursos de rede de negócios e recursos institucionais (Prim, Amal \& Carvalho, 2016). Enquanto os recursos locais incluem profissionais, infraestrutura e mercado local; os recursos da rede de negócios constituem os stakeholders envolvidos economicamente no cluster, como 
fornecedores, clientes, concorrentes e empresas relacionadas; e os recursos institucionais representam organizações educacionais, profissionais e organizações de governança do cluster, aqui denominadas, organizações de apoio (Prim, Amal \& Carvalho, 2016).

Considerando as afirmativas sobre cluster, estratégias, tecnologias e recursos, afirma-se a presença, participação e importância das organizações de apoio (Maia, Antunes \& de Castro, 2019), reconhecidas como recursos institucionais do cluster, que de forma direta ou indireta, passam a auxiliar ou mesmo exercer a governança do arranjo (Bocquet et al., 2016). A governança em cluster está relacionada a mecanismos de influência que buscam maximizar a competitividade das empresas por meio de ações que minimizem conflitos e promovam estratégias que favoreçam o aglomerado (Arikan \& Schilling, 2011; Lefebvre, 2016).

Neste cenário, a capacidade de formulação e implantação de estratégias de resultado orientadas para o cluster, criadas com a intenção de beneficiar as empresas do aglomerado, pode ser apoiada pela existência e atuação de organizações de apoio localmente estabelecidas (Pereira et al. 2014). Do mesmo modo, a introdução de tecnologias pode ser potencializada a partir da existência de organizações de apoio à pesquisa e desenvolvimento tecnológico que atuem no cluster (Sarturi et al., 2016), que estimulam a criação e a troca de conhecimentos (Arikan, 2009). Todos esses movimentos, processos e ações das organizações envolvidas relaciona-se com a governança do cluster.

\section{Governança no contexto dos clusters}

A expressão governança apresenta distintos significados dependendo dos princípios assumidos, da forma de construção dos mecanismos e da valorização de algum fator mais determinante (Roth et al., 2012). Alguns autores valorizam a racionalidade e formalidade das regras de ações coletivas (Provan \& Kenis, 2008; Wallenburg \& Schäffler, 2014), outros focam na estrutura dos papéis e funções dos atores (Gorriz-Mifsud et al. 2017), outros ainda buscam unir aspectos racionais, explícitos e contratuais, com aspectos relacionais (Grandori, 2006; Peng, Tu, Wei, 2018). Cada linha de pensamento está associada a alguns aspectos de ações em rede, como regras, papéis, hierarquia, práticas de produção, valores compartilhados (Roth et al., 2012).

Apesar da variedade de abordagens, há convergência na afirmativa que a governança se refere aos mecanismos criados e seguidos num grupo, os quais facilitam as ações coletivas, por exemplo, sobre os modos de produção, de controles do comportamento e modos de decisão sobre os problemas e oportunidades (Grandori, 2006; Moeliono et al., 2016; Angst \& Hirschi, 2017; Gottschick, 2018; Kellogg \& Samanta, 2018; Partelow \& Nelson, 2020).

Conforme revisão conceitual realizada pelos autores do presente artigo, essa convergência sobre o que é e qual a função da governança já aparecia em teorias econômicas tradicionais sobre redes (Williamson, 1979, 1996), teorias estruturais de redes (Borgatti et al., 2009), abordagens sociais, de construção gradativa dos mecanismos (Jones, Hesterly \& Borgatti, 1997), ou de imersão e 
comprometimento dos atores (Granovetter, 1985). Em todas essas vertentes se coloca que mesmo que exista uma governança formal claramente estabelecida, por exemplo, um contrato bem detalhado, as partes sempre precisam conversar e ajustar os mecanismos. Isto ocorre porque nenhum contrato é capaz de cobrir todas as situações possíveis e imprevisíveis de uma tarefa em grupo (Chen, Lin \& Wang, 2018).

Essa interface entre a governança formal e a governança construída (também chamada de relacional, colaborativa ou informal) é tema constante de pesquisa, porque se refere ao cerne do desenvolvimento de recursos competitivos de uma rede. Temas como divergência de cultura (Ibert \& Muller, 2015); assimetrias de capacidades (Hahl, Kacperczyk \& Davis, 2016); modos de resolver tarefas (Paul, 2012); associação entre estrutura e relacionamento (Miele \& Matias, 2017); imersão estrutural (Kim \& Jin, 2017) e performance (Niesten et al., 2017) são exemplos da importância e necessidade de uma governança construída entre os atores. Conforme Cropper et al. (2008), Kathleen, Michael e Brian (2009) e Ouro Filho, Olave e Barreto (2015) os atores de uma rede buscam coesão, eliminação de conflitos e eficiência produtiva.

Esse processo de construção da governança, na busca da competitividade do grupo, pode ser aplicado aos clusters, pois as empresas que fazem parte do clusters compartilham ligações verticais e horizontais, de natureza formal e informal, com o maior número possível de participantes, buscando sua eficiência (Todeva, 2006). As organizações de apoio estão contempladas nesse conceito de processo coletivo.

Tal como em outros formatos de redes, nos clusters também estão presentes os elementos de assimetria de interesses e de capacidades, ao lado da necessidade de tarefas conjuntas, o que exige a construção e presença da governança (Ingstrup \& Damgaard, 2013; Bocquet et al., 2016). Nos clusters, a governança surge para minimizar conflitos de interesse, de distribuição de poder e de lealdade entre os atores, uma vez que, devido à interdependência existente, não raro os esforços de um ator se sobrepõem aos de outro, impedindo que ambos atinjam seus objetivos (Van Aken \& Weggeman, 2000).

Nessa linha conceitual, a análise da governança de um cluster pode valorizar os processos sociais de construção dos mecanismos, incluindo as organizações de apoio, estejam eles em status formalizados, ou não (Poppo \& Zenger, 2002). Em artigo clássico, Dyer e Singh (1998, p.669) afirmaram que a governança relacional, no sentido de construção dos mecanismos, é importante para o controle dos custos, do comportamento de comprometimento dos atores e dos modos de decisão de tarefas. O mesmo raciocínio foi apresentado em estudos posteriores (Gulati, Nohria \& Zaheer., 2000; Zaccarelli et al., 2008).

Para caracterizar a governança de um cluster local, Bolumole, Closs e Rodammer (2015) cunharam a expressão governança regional, que contém os mesmos processos já descritos, somados aos recursos específicos do local. Neste trabalho, adota-se o conceito de governança como construção social, aceita-se sua relação de consequência na produtividade e competitividade de um grupo e, adota-se o conceito de governança regional, caracterizando uma conjunção local de organizações, sejam 
relacionadas diretamente à tarefa, sejam de organizações de apoio como, por exemplo, universidades e associações.

Os conceitos descritos oferecem uma base competente para a investigação da participação das organizações de apoio nos clusters. A Figura 1 apresenta o resumo dos conceitos e sua ligação com a pergunta orientadora.

Figura 1 - Matriz conceitual e a relação com o tema das organizações de apoio

\begin{tabular}{|c|c|c|}
\hline Conceito & Resumo do Conceito & Ligação com questão orientadora \\
\hline Clusters & $\begin{array}{l}\text { Aglomerações geográficas de empresas, } \\
\text { fornecedores, prestadores de serviços e } \\
\text { instituições associadas em um campo } \\
\text { específico, vinculado por externalidades e } \\
\text { complementaridades de vários tipos. }\end{array}$ & $\begin{array}{l}\text { Organizações de apoio estão no } \\
\text { mesmo nível de importância das } \\
\text { outras na realização da tarefa. }\end{array}$ \\
\hline Competitividade & $\begin{array}{l}\text { Habilidade de produzir bens e serviços no } \\
\text { tempo, local e forma desejados pelos } \\
\text { consumidores, a preços melhores ou } \\
\text { equivalentes aos potenciais concorrentes, } \\
\text { sendo estes preços suficientes para ao menos, } \\
\text { remunerar o custo de oportunidade dos } \\
\text { recursos empregados. Considerando a } \\
\text { competitividade de grupos, fatores tais como } \\
\text { inovação coletiva e compartilhamento de } \\
\text { recursos tornam-se importantes. }\end{array}$ & $\begin{array}{l}\text { Organizações de apoio incrementam a } \\
\text { competitividade do cluster porque são } \\
\text { capazes de gerar recursos de } \\
\text { inovação, recursos tecnológicos e de } \\
\text { capital social, no sentido de uma rede } \\
\text { ampla que se pode acessar quando um } \\
\text { recurso é necessário. }\end{array}$ \\
\hline Governança & $\begin{array}{l}\text { Conjunto de mecanismos, práticas, normas, } \\
\text { regras que incentivam as ações coletivas e } \\
\text { controlam o comportamento dos atores. }\end{array}$ & $\begin{array}{l}\text { Como pressuposto, todas as } \\
\text { organizações participantes de um } \\
\text { clusters estão envolvidas na formação } \\
\text { e ajustes dos mecanismos que } \\
\text { compõem a governança, incluindo as } \\
\text { organizações de apoio. }\end{array}$ \\
\hline Governança regional & $\begin{array}{l}\text { Quando o processo de governança se realiza } \\
\text { num local geográfico delimitado, com } \\
\text { características marcantes de construção dos } \\
\text { mecanismos realizada pelos próprios atores. }\end{array}$ & $\begin{array}{l}\text { O conceito afirma que as } \\
\text { organizações locais, incluindo as } \\
\text { organizações de apoio, são as que } \\
\text { constroem e implementam a } \\
\text { governança e, como consequência, } \\
\text { nos resultados do cluster. }\end{array}$ \\
\hline Pergunta orientadora & $\begin{array}{l}\text { Como participam e qual a importância das } \\
\text { organizações de apoio na competitividade de } \\
\text { um cluster? }\end{array}$ & $\begin{array}{l}\text { Considerando os conceitos de cluster, } \\
\text { competitividade e governança, } \\
\text { objetiva-se avançar no conhecimento } \\
\text { da questão colocada, já que existem } \\
\text { poucos trabalhos empíricos sobre o } \\
\text { tema. }\end{array}$ \\
\hline
\end{tabular}

Fonte: Elaborada pelos autores.

Considerando o fato da raridade de pesquisas e exemplos de como as organizações de apoio participam nos clusters (Ingstrup \& Damgaard, 2013; Bocquet et al., 2016), define-se o objetivo do artigo em apresentar detalhes das ações e das linhas de influência de organizações de apoio em dois clusters, a partir das lentes da governança e da competitividade. $\mathrm{O}$ artigo, portanto, tem um potencial de inovatividade ao lançar o foco nas organizações de apoio e um grau de ineditismo ao apresentar as 
categorias de análise comparativa entre clusters, o que ainda não havia se encontrado na literatura voltada ao papel das organizações de apoio.

\section{Procedimentos metodológicos}

Em diferentes áreas de estudos de gestão, como comportamento organizacional e estratégia, a pesquisa indutiva frequentemente requer métodos qualitativos de pesquisa, como estudos de caso, método adotado na presente pesquisa, que permite responder questões "como" e "por que", (Woiceshyn e Daellenbach, 2018; Eriksson e Kovalainen, 2016, p. 42). Esse trabalho busca entender como as organizações de apoio atuam sobre a governança e sua influência na competitividade de clusters de negócios. Trata-se de uma pesquisa de natureza qualitativa e método indutivo.

A indução diz respeito à generalização dos resultados a partir das observações disponíveis, a partir do raciocínio do pesquisador na observação e organização de padrões nos dados (Woo, O'Boylee Spector, 2017). A pesquisa indutiva busca detectar fatores organizacionais e processos em cortes transversais e temporais (Woiceshyn e Daellenbach, 2018; Eriksson e Kovalainen, 2016, p. 42), num trabalho de busca por padrões, similaridades e diferenças. O método indutivo supõe que a constatação de padrões em vários casos possibilita a quebra de explicações simplistas, isto é, descritivas de um único caso, incrementando a compreensão do fenômeno (Eisenhardt, 1989).

Para alcançar o objetivo por meio do método indutivo, esta pesquisa realiza um estudo de caso múltiplo adotando cross-case display, ou seja, ao se observar diferentes casos busca-se entender como eles são qualificados pelas condições locais, nas suas convergências e diferenças, e, assim, aprofundar a compreensão e explicação do fenômeno por meio de descrições mais sofisticadas e explicações mais elaboradas, que transcendem a descrição de caso único (Miles \& Huberman, 1994).

Para a construção inferencial que caracteriza o método indutivo, o presente trabalho utiliza a metodologia de construção de categorias de análise (Bardin, 2009), a partir dos dados de fontes primárias, como discursos, e secundárias, como atas de reuniões, ou contratos. O foco da análise está centrado nas evidências de participação das organizações de apoio locais sobre a governança, as estratégias e a competitividade do cluster.

Todo o processo de indução foi sustentado na estratégia de construção das categorias de análise conforme emergiram dos dados. Esse caminho de construção gradativa das categorias oferece sustentação na indução e nas interpretações, já que segue o caminho da empiria para o teórico. $\mathrm{O}$ software NVIVO foi utilizado para organizar os discursos em conceitos emergentes e, a partir destes construir as categorias que agregam esses conceitos.

\section{Casos estudados}

Nesta pesquisa, analisamos dois clusters da área de ciências da saúde: o Cluster da Saúde de Ribeirão Preto - SP, no Brasil, e Oxfordshire Health Science Cluster, na Inglaterra. Ambos os casos 
(clusters) são reconhecidamente competitivos e fazem parte de regiões altamente concentradas no setor de ciências da saúde: produzem medicamentos, cosméticos, equipamentos médicos, proveem serviços médicos, odontológicos e veterinários, e atividades correlatas.

A opção por esses casos se justifica por quatro razões: (i) ambos os clusters apresentam alta concentração de empresas que operam no mesmo setor; (ii) ambos os casos são referência em relação à competitividade no setor em seus países; (iii) tratam-se de casos contrastantes considerando as condições sociais e econômicas distintas do país de origem; (iv) ambos contam com a presença de universidades reconhecidas por influenciar o desenvolvimento tecnológico do setor da saúde por meio de incubadoras, agências de inovação, grupos de pesquisa, etc.

\section{Coleta de dados}

Foram coletados dados de múltiplas fontes; os dados de fontes secundárias incluem relatórios empresariais, notícias, e webpages de organizações relacionadas aos clusters. Também foram coletados dados de fontes primárias, por meio de observações de campo e, principalmente, entrevistas semiestruturadas, utilizadas, conforme Gioia, Corley e Hamilton (2013), para captar informações passadas e presentes acerca do fenômeno estudado.

No método proposto, as entrevistas semiestruturadas foram realizadas com especialistas pertencentes aos clusters. O conceito de especialista se refere ao indivíduo que tem especial conhecimento sobre o fenômeno estudado e está de acordo com conceito de informante chave proposto por Fetterman (1998, p.483).

Um protocolo de entrevistas foi criado previamente com base na literatura sobre o tema (Eisenhardt, 1989) e em uma etapa preliminar da coleta e análise de dados de fontes secundárias, especialmente os coletados em webpages de organizações pertencentes aos clusters. Em fevereiro de 2015 foi realizado o pré-teste do roteiro de entrevistas em um Research Park na região Sudeste da Inglaterra, onde atuam empresas de ciências da saúde.

Nesta pesquisa, optou-se pela seleção intencional de respondentes, buscando-se aqueles que atendem aos critérios de interesse e representam variedade dentro desses critérios (Patton, 1990). Com o intuito de permitir uma comparação adequada, selecionaram-se entrevistados de organizações e funções semelhantes para os dois casos: Agência de Inovação; Associações Empresariais; Centro de Pesquisa; Grupos de pesquisa universidade - empresa; Network Center; Redes Acadêmicas; e serviços de suporte.

A fim de preservar a identidade dos entrevistados foi mantido sigilo sobre nomes e cargos. Salienta-se que os entrevistados expressaram suas opiniões pessoais embora sigam as diretrizes das empresas e não tenham relatado informações confidenciais sobre as organizações. As entrevistas foram realizadas no período de março a setembro de 2015 com membros das organizações de apoio 
identificadas nessa pesquisa, ao todo foram realizadas 6 entrevistas no cluster de Ribeirão Preto e 7 entrevistas no cluster de Oxfordshire. Conforme detalhado na Figura 2.

Figura 2 - Especialistas entrevistados

\begin{tabular}{|c|c|c|}
\hline País & Organização & Cargo \\
\hline \multirow{6}{*}{ Brasil } & Agência USP de Inovação & Agente de Inovação \\
\hline & $\begin{array}{l}\text { Instituto Nacional de Ciência e Tecnologia para } \\
\text { Inovação Farmacêutica (INCT-IF). }\end{array}$ & Coordenador \\
\hline & $\begin{array}{l}\text { Arranjo Produtivo Local de Saúde - APL de } \\
\text { Saúde }\end{array}$ & $\begin{array}{l}\text { Diretor executivo, diretor técnico e } \\
\text { empreendedor membro do APL. }\end{array}$ \\
\hline & $\begin{array}{l}\text { Fundação Instituto Pólo Avançado da Saúde de } \\
\text { Ribeirão Preto (FIPASE) }\end{array}$ & $\begin{array}{l}\text { Gerente de Desenvolvimento Econômico e } \\
\text { Tecnológico }\end{array}$ \\
\hline & $\begin{array}{l}\text { Serviço Brasileiro de Apoio as Micro e } \\
\text { Pequenas Empresas (SEBRAE-RP) }\end{array}$ & $\begin{array}{l}\text { Consultor Especialista em Gestão da } \\
\text { Inovação }\end{array}$ \\
\hline & $\begin{array}{l}\text { Research Center on Innovation, Technology } \\
\text { Management and Competitiveness (InGTeC) }\end{array}$ & Coordenador \\
\hline \multirow{7}{*}{ Inglaterra } & Isis Innovation & $\begin{array}{l}\text { Deputy Head of Technology Transfer - } \\
\text { Pharma and Biotech }\end{array}$ \\
\hline & $\begin{array}{l}\text { Oxford Academic Health Science Center } \\
\text { (OAHSC) }\end{array}$ & Director of Commercial Development \\
\hline & Oxford Business Network $(O B N)$ & Analyst and EU Projects Manager \\
\hline & NIHR Biomedical Research Centre & BRC Project Officer \\
\hline & $\begin{array}{l}\text { South East Health Technologies Alliance } \\
\text { (SEHTA) }\end{array}$ & Chief Executive \\
\hline & $\begin{array}{l}\text { Oxford Institute of Biomedical Engineering } \\
(I B M E)\end{array}$ & Director and cluster's entrepreneur \\
\hline & Oxfordshire Economic Observatory & Founder and Director of Research \\
\hline
\end{tabular}

Fonte: Dados da pesquisa.

Ressalta-se que a quantidade de organizações e entrevistados foi determinada de modo a potencializar o valor das informações obtidas e a compreensão acerca do assunto até atingir a saturação. Outras organizações de apoio estão presentes nos clusters, por exemplo, outros grupos de pesquisa das universidades da região. Porém, optamos pelas que foram mais citadas em artigos, sites, notícias e por indicação dos próprios entrevistados.

\section{Procedimento de análise}

As entrevistas foram transcritas, lidas e submetidas ao software NVIVO, para serem codificadas em categorias que ajudassem a explicar como as organizações de apoio atuam sobre a governança e sua consequente influência na competitividade de clusters. O uso do software implicou em realizar algumas rodadas de análise dos dados. Nas primeiras rodadas, foram criadas categorias descritivas e, nas rodadas seguintes, categorias relacionadas à literatura. O uso do software NVIVO permitiu que após a codificação o texto sob certa categoria fosse visto em um único documento, o que facilitou o refinamento 
dos dados. Resumos foram gerados para cada categoria, o que permitiu contrastar os dados com a literatura que fundamenta a pesquisa.

As análises seguiram os seguintes passos: seleção, transformação, codificação e agregação dos dados brutos, conforme o método proposto por Miles e Huberman (1994). Tanto as anotações de campo, transcrições de entrevistas, relatórios e outros materiais, foram analisados em conformidade com estes critérios.

A Figura 3 ilustra o caminho percorrido, para se chegar na categoria "Captar e direcionar recursos". O itálico indica os pontos chave destacados pelo software.

Figura 3 - Ilustração do caminho de construção das categorias em dois clusters

\begin{tabular}{|c|c|c|}
\hline $\begin{array}{c}1^{\mathrm{a}} \text { Ordem } \\
\text { Conceito emergente }\end{array}$ & $\begin{array}{c}2^{\mathrm{a}} \text { Ordem } \\
\text { Conceito emergente }\end{array}$ & $\begin{array}{c}\text { Agregação - } \\
\text { Papel das organizações } \\
\text { de apoio }\end{array}$ \\
\hline $\begin{array}{l}\text { Existem programas na Secretaria de Desenvolvimento } \\
\text { do Estado de São Paulo, que tem recursos específicos } \\
\text { para APLs, então é possível também captar esses } \\
\text { recursos para trazer infraestruturas para as cidades, } \\
\text { para a região, isso é importantíssimo também, só as } \\
\text { empresas organizadas em grupo conseguirão buscar } \\
\text { esses recursos (Entrevistado RP-4). }\end{array}$ & $\begin{array}{l}\text { Concorrer em editais de } \\
\text { programas do governo } \\
\text { específicos para destinar } \\
\text { recursos a clusters } \\
\text { (Arranjos Produtivos } \\
\text { Locais) }\end{array}$ & \multirow{2}{*}{$\begin{array}{c}\text { Captar e direcionar } \\
\text { recursos }\end{array}$} \\
\hline $\begin{array}{l}\text { So, the sort of things that get debated at the AHSC are } \\
\text { global, are non-scale things, like how do we present } \\
\text { the cluster better to the outside world? Is there } \\
\text { enough... what are the issues that are holding back the } \\
\text { cluster? So it could be something like a transport } \\
\text { infrastructure or lack of premises for companies. [...] } \\
\text { They (the AHSC) will try and present arguments to } \\
\text { people who have money for infrastructure projects } \\
\text { (Entrevistado Oxf-7). }\end{array}$ & $\begin{array}{l}\text { Argumentar com pessoas } \\
\text { que têm dinheiro para } \\
\text { investir nos clusters, por } \\
\text { exemplo, angels. }\end{array}$ & \\
\hline
\end{tabular}

Fonte: Dados da pesquisa.

Análise e discussão dos achados

\section{Presença de organizações de apoio e sua influência na governança dos clusters}

Tanto no cluster de Oxford quanto no cluster de Ribeirão Preto foi constatada a presença de tipos diferentes de organizações de apoio que atuam no desenvolvimento da região e exercem influência na governança, estratégia e competitividade dos clusters. Dentre as organizações, encontram-se algumas relacionadas às universidades, o que está de acordo com o seu papel na formação e crescimento dos clusters.

No entanto, conforme ficou evidenciado nos discursos, a simples existência de organizações com a finalidade de prestar algum suporte e apoio ao cluster não garante seu êxito e adesão das empresas ali inseridas, sendo necessário que elas gerem valor para as outras organizações, pois só assim serão 
reconhecidas e terão poder de influência. Algumas organizações de apoio encontradas nos clusters pesquisados, incluindo exemplos desse tipo de networks, estão relacionadas na Figura 4.

Figura 4 - Organizações de apoio presentes nos clusters

\begin{tabular}{|c|c|c|}
\hline $\begin{array}{c}\text { Tipo de } \\
\text { organização }\end{array}$ & Ribeirão Preto & Oxfordshire \\
\hline \multirow{3}{*}{$\begin{array}{l}\text { Organizações } \\
\text { diretamente } \\
\text { relacionadas às } \\
\text { empresas }\end{array}$} & $\begin{array}{l}\text { Fundação Polo Avançado da Saúde } \\
\text { (FIPASE) }\end{array}$ & $\begin{array}{l}\text { Oxford Academic Health Science Center } \\
\text { (OAHSC) }\end{array}$ \\
\hline & APL da Saúde (Associação) & Oxford Business Network (OBN) \\
\hline & $\begin{array}{l}\text { Serviço de Apoio às Micro e Pequenas } \\
\text { Empresas de Ribeirão Preto (SEBRAE-RP) }\end{array}$ & $\begin{array}{l}\text { Oxford Academic Health Science Network } \\
\text { (OAHSN) }\end{array}$ \\
\hline \multirow{4}{*}{$\begin{array}{l}\text { Organizações } \\
\text { relacionadas à } \\
\text { pesquisa }\end{array}$} & Agência USP de Inovação & Isis Innovation \\
\hline & $\begin{array}{l}\text { Instituto Nacional de Ciência e Tecnologia } \\
\text { para inovação farmacêutica (INCT-if) }\end{array}$ & $\begin{array}{l}\text { Oxford Institute of Biomedical Engineering } \\
\text { (IBME) }\end{array}$ \\
\hline & $\begin{array}{l}\text { Núcleo de Pesquisas em Inovação, Gestão } \\
\text { Tecnológica e Competitividade (InGTeC) }\end{array}$ & $\begin{array}{l}\text { Biomedical Research Centre (BRC) - } \\
\text { National Institute for Health Research } \\
\text { (NIHR) }\end{array}$ \\
\hline & & Oxfordshire Economic Observatory \\
\hline
\end{tabular}

Fonte: Dados da pesquisa.

A governança é vista pelos entrevistados como algo importante para que haja conexão nos clusters e desenvolvimento da região, conforme ilustra o excerto a seguir:

"Existia uma cadeia produtiva [em Ribeirão Preto], uma concentração significativa de empresas e uma governança estabelecida, então, entendeu-se que a associação APL da Saúde poderia apoiar essas empresas como na forma de um cluster, dado a sua importância para o desenvolvimento regional e territorial" (Entrevistado RP-4).

Os entrevistados notaram um aumento da presença da governança no decorrer do tempo. Porém, não a veem como algo a ponto de impor regras ou mudanças às empresas, mas sim como algo capaz de ajudar na criação de um ambiente mais adequado para o desenvolvimento destas empresas. Observouse que os entrevistados percebem que a governança é exercida por organizações que apoiam o cluster. Nas seções a seguir serão analisadas algumas das atividades desenvolvidas por estas organizações de apoio presentes nos clusters estudados.

\section{Atividades desempenhadas pelas organizações de apoio}

Os dados evidenciaram exemplos de como as organizações de apoio podem se comportar como agentes de ações de governança nos clusters. Ao analisar a fala dos entrevistados, nota-se que são depositadas nas organizações de apoio, expectativas de resolução de problemas. Um exemplo são as atividades que relacionadas à bens públicos ou de uso comum nos clusters, encontradas nos discursos dos dois casos, conforme ilustração: 


\begin{abstract}
"What are the issues that are holding back the cluster? It could be something like a transport infrastructure or lack of premises for companies. [...] They [AHSC] will try and present arguments to people who have money for infrastructure projects" (Entrevistado Oxf-7).
\end{abstract}

"existem programas na Secretaria de Desenvolvimento do Estado de São Paulo, que tem recursos específicos para APLs. É possível captar esses recursos para trazer infraestruturas para a região, $[\ldots]$ e e só as empresas organizadas em grupo conseguirão buscar esses recursos" (Entrevistado RP-4).

Dentre as atividades desempenhadas pelas organizações de apoio, fomentar uma cultura de participação e colaboração entre as empresas é uma das atividades tidas como mais relevantes, mas que não é tarefa fácil, conforme relato a seguir:

"tem hora que começa a diminuir a participação aí a gente vai lá chama todo mundo, faz uma reunião, começa de novo. Porque as empresas além de participar do APL da Saúde (associação) têm que produzir e vender, então muitas vezes deixam de ir para poder trabalhar" (Entrevistado RP-5).

O Entrevistado Oxf-6 refletindo sobre as organizações de apoio nos cluster fala sobre a importância de pessoas, ou organizações com know-how para ajudar as empresas:

"it adds collectivity, if people needs some advice, if you grow a business, you always need advice, you always are going to a new step where you never been before... there are always people that you can consult on that. Or if that person can't help they will know somebody who does" (Entrevistado Oxf-6).

Os discursos também se reportam à outras ações das organizações de apoio, que beneficiam o cluster, tais como recursos humanos e materiais:

"One of those benefits may be working together to make a new product, or quite a range of service providers, specialized consultants who have, for example, experience in clinical trials or formulations or pharmaceutical aspects or you know, that kind of specialist knowledge" (Entrevistado Oxf-7).

As organizações muitas vezes colaboram entre si para promover ações e atrair outros parceiros. Como exemplo, o Entrevistado RP-4 relatou uma parceria entre o SEBRAE, o APL da Saúde e o Banco Interamericano de Desenvolvimento (BID), em que o SEBRAE promoveu Fóruns de Competitividade, onde se convidam todos os atores envolvidos, as empresas, as indústrias, os operadores de saúde, os profissionais, e promoviam-se discussões técnicas.

No cluster de Oxfordshire podem-se citar ações em conjunto realizadas pela OBN e pela OAHSN, organização voltada para as ciências da vida. O resultado desta ação foi um mapa do cluster de ciências da saúde daquela região, com acesso livre no website da OAHSN e que contém informações sobre o setor. 
Outro tipo de ação estratégica para o cluster são as reivindicações junto ao poder público em favor das empresas do aglomerado. Em Ribeirão Preto, além da conquista de um laboratório pago e instalado pelo poder público, a FIPASE entrou em contato com a Secretaria de Desenvolvimento do Estado de São Paulo para reivindicar que os cursos oferecidos pela FATEC de Ribeirão Preto atendessem às necessidades de mão de obra especializada para o cluster. Outro exemplo é o que o APL da Saúde faz em relação às normatizações estabelecidas pela ANVISA, representando as empresas nos diálogos com esta instituição. O mesmo discurso de lobby foi apresentado pelos entrevistados da Inglaterra.

Em resumo, conforme analisado, ambos os clusters apresentam indícios da presença de governança e da existência do fundamento "Estratégia de resultado orientada para o cluster". Em ambos os casos, existe certa diversidade de organizações que apoiam as empresas e que desenvolvem ações em favor da melhoria da competitividade da entidade supra empresarial. Os dados sustentam a proposição do artigo, sobre a importância e participação das organizações de apoio na estratégia do cluster e se alinha com afirmativas teóricas descritas em item anterior.

\section{Atividades relacionadas especificamente à inovação desempenhadas pelas organizações de apoio}

Por meio de ações como a desenvolvida pela Agência USP de Inovação, chamada Programa Vocação para a Inovação, as organizações de apoio estimulam a competitividade dos clusters. Outros exemplos são verificados nos grupos de pesquisa como o InGTeC que promovem o Seminário de Rotas Tecnológicas, realizado em parceria com a FIPASE, um seminário nacional que acontece em Ribeirão Preto. Essas ações obtêm como um dos resultados a ampliação de participação de empresários locais.

As organizações de apoio à inovação, identificadas em ambos os clusters, são agências de inovação e incubadoras de empresas. O cluster de Ribeirão Preto conta com a presença da Agência USP de Inovação, que é o Núcleo de Inovação Tecnológica da Universidade de São Paulo. Essa agência é responsável por gerir a política de inovação para promover a utilização do conhecimento científico, tecnológico e cultural produzido na universidade (USP INOVAÇÃO, 2016). No cluster de Oxfordshire, encontra-se a Isis Innovation, empresa $100 \%$ pertencente à University of Oxford e que comercializa a pesquisa e tecnologia desenvolvida por esta universidade (ISIS INNOVATION, 2016). Essas duas agências desempenham funções similares em seus respectivos clusters.

Inicialmente ambas as organizações foram criadas com o intuito de facilitar a transferência de tecnologias desenvolvidas dentro das universidades por professores, pós-graduandos e outros pesquisadores. Conforme essas organizações foram evoluindo, foi gerada uma demanda por seus serviços por parte de outras empresas da região. Para atender a esta demanda a Isis Innovation criou $a$ Isis Enterprise, que é essencialmente um grupo de consultoria em negócios e a Agência USP de Inovação criou o Programa Vocação para a Inovação - Apoio à Propriedade Intelectual para o Estado de São Paulo. 
À medida que sua atuação extrapolou os limites da universidade, essas organizações de apoio passaram a desempenhar papéis relevantes para a governança dos clusters. Ao entender governança como o exercício de influência orientadora, percebe-se a presença de governança tanto no aspecto relacional, ao auxiliar empresários em relações interpessoais facilitando a comunicação e parcerias, e formal, nas negociações e elaboração de contratos entre empresários e entre empresários e investidores.

Sobre propriedade intelectual, os sujeitos relataram a ação da Agência USP de Inovação e da FIPASE, buscando parcerias com consultorias renomadas para auxiliarem as empresas do cluster. Esse é um fato relevante, porque uma forma de analisar o caráter evolucionário de um cluster de tecnologia é verificar se há a presença de startups e spin offs, empresas que, em geral se desenvolvem em incubadoras e parques tecnológicos (Gagné et al., 2010). No cluster brasileiro, encontra-se a Supera Incubadora de Empresas de Base Tecnológica, localizada no Parque Tecnológico de Ribeirão Preto. No cluster britânico, encontram-se a Isis Startup Incubator, Oxford University's Science Park em Begbroke, além de Harwell e Milton Park.

Startups e spin offs são empresas voltadas diretamente para a descoberta de novos produtos e novos processos e que devido ao seu caráter inovador geram impacto direto no avanço da tecnologia das demais empresas do cluster. De forma simples, spin outs normalmente envolvem propriedade intelectual, enquanto startups não necessariamente envolvem propriedade intelectual, mas sim uma ideia que pode criar um novo negócio.

Apesar do elevado custo do espaço, ressaltado por alguns entrevistados, o número dessas empresas está aumentando e há ainda potencial para crescimento em ambos os clusters, conforme trechos a seguir:

"há um número cada vez maior de startups [...]. E se continuar nesse ritmo, talvez daqui uns 20 anos tenha um número maior de empresas inovadoras do que não inovadoras". (Entrevistado RP-1).

"there a lot of companies effectively spinning out other companies as well. And that it is like a snowball, it potentially can grow and grow" (Entrevistado Oxf-1).

Concluindo este subitem, fica claro que ambos os clusters apresentam evidências de que estão evoluindo através da introdução de tecnologias e que em ambos o fenômeno ocorre, em parte, pela participação de organizações de apoio. Os efeitos são o desenvolvimento de tecnologias, a troca de informação, o desenvolvimento e o aumento do número de startups e spin offs nos clusters. Tudo girando ao redor de uma governança participativa gerenciada pelos próprios atores do cluster.

\section{Compilação das Funções Desempenhadas pelas Organizações de Apoio}

Após analisar os dados emergentes das entrevistas, observações e outros materiais, foi possível realizar um compilado que sintetiza as frentes de atuação das instituições de apoio nos clusters, e os 
desdobramentos das atividades por elas desempenhadas em prol da orientação estratégica do cluster e inovação por introdução de tecnologias. O resultado está apresentado na Figura 5, em duas colunas, com a da esquerda apresentando a análise final dos conceitos emergentes, para se chegar na agregação dos conceitos, na coluna da direita.

Figura 5 - Conceitos emergentes e conceitos agregados

\begin{tabular}{c|c} 
Conceito emergente & $\begin{array}{c}\text { Agregação dos conceitos: } \\
\text { Papel das organizações de apoio }\end{array}$ \\
\hline
\end{tabular}

Concorrer em editais de programas do governo específicos para destinar recursos a clusters (Arranjos Produtivos Locais).

Argumentar com pessoas que têm recursos financeiros para investir nos clusters, por exemplo, angels.

\section{Captar e direcionar recursos}

Grupo para poder discutir normas, leis que afetam o setor.

Grupo de compras coletivas de itens em comum para as empresas.

Consórcio para negociação de descontos e benefícios com fornecedores em comum.

Promover a colaboração entre as empresas por meio da formação de grupos

Frente de advocacia negociando impostos e investimentos.

Organização de sessões de negócio, encontros, feiras.

Promover eventos para criação de redes e de parcerias entre empresários e fornecedores.

Organizar eventos para criação de redes e de parcerias entre empresários, acadêmicos e investidores.

Promover eventos que

proporcionam um ambiente para network entre as empresas do cluster

Organização de workshops, treinamento.

Providenciar elaboração de diagnóstico, mapeamento das tendências, rotas tecnológicas.

Reunir os membros para ouvir sobre um tema interessante ou apresentar suas empresas.

\section{Auxiliar o desenvolvimento de competências no cluster}


Agregação dos conceitos:

Papel das organizações de apoio

Reivindicar ao poder público demandas das empresas do setor, como instalação de laboratórios.

Realização de lobby (legal) por órgãos de comércio em favor das empresas em relação às leis, impostos, legislação ou exportação.
Auxiliar os pesquisadores a realizar patente, de registro de software, de marca, direitos autorais, desenho industrial.

Auxiliar na criação de empresas com propriedade intelectual, encontrar um CEO, elaborar plano de negócios, ajudar a garantir o investimento.

Auxiliar os pesquisadores nos processos de negociação de licenciamentos, na negociação e nos convênios colaborativos com empresas.

Licenciamento de tecnologias na indústria, e licenciamento para de spin outs.

Fomentar a inovação e empreendedorismo e ser responsável pelo contato e gestão com incubadoras e parques tecnológicos.

Incubar empresas gerando spin-offs em que a universidade se torna acionista com os acadêmicos fundadores e os investidores.

\section{Representação junto ao poder público}

Auxiliar nos processos de proteção e negociação do conhecimento ou tecnologia gerada
Auxiliar a criação de novos negócios por meio de incubadoras

Fonte: Dados da pesquisa.

A descrição mais detalhada de cada um dos extratos é apresentada a seguir.

\section{A1. Captar e direcionar recursos.}

Os entrevistados revelaram: (i) uma atuação contínua por parte das instituições de apoio, visando a formação de grupos para discutir normas e legislações que afetam o setor de atuação dos clusters, além de (ii) coletivamente buscarem por vias jurídicas negociar impostos e investimentos. Além dessas atividades, também fomentam a comercialização e otimização de custos mediante (iii) a formação de grupos para compras coletivas e (iv) formação de consórcios de compra para descontos e benefícios com fornecedores comuns.

Também foi possível observar uma atuação constante por parte das entidades de apoio em (i) organizar sessões de negócios, encontros e feiras, (ii) promover eventos para criação de redes e de 
parcerias entre as empresas locais e seus fornecedores, (iii) bem como para criação de redes e de parcerias entre empresários, acadêmicos e investidores, (iv) além de buscar a capacitação dos atores com a organização de workshops e treinamentos.

Considerando as ações mencionadas, entende-se que ao promover a integração dos atores do cluster em prol de objetivos coletivos comuns, captando e direcionando recursos, resulta que uma das atividades das instituições de apoio no direcionamento estratégico é:

A2. Promover a colaboração entre as empresas por meio da geração de um ambiente integrador $e$ formação de grupos.

As instituições de apoio também são proeminentes em (i) elaborar diagnósticos, mapear tendências e rotas tecnológicas no setor de atividade do cluster, e em levar estas informações aos membros (ii) reunindo-os para ouvir sobre um tema de interesse comum e que lhes possa ser útil. Dessa forma, percebe-se que as instituições de apoio atuam estrategicamente ao:

\section{A3. Auxiliar o desenvolvimento de competências no cluster.}

Outro importante papel desempenhado pelas instituições de apoio a governança dos clusters envolve a representatividade que essas instituições desempenham perante o poder público, seja ao (i) reivindicar demandas das empresas do setor, como por exemplo a instalação de laboratórios, ou ainda (ii) a realização de lobby (legal) por órgãos de comércio em favor das empresas em relação às leis, impostos, legislação ou exportação. Essa frente de atuação pode ser resumida na atividade:

\section{A4. Exercer representação em nome do cluster junto ao poder público}

As instituições de apoio também participam da governança perseguindo a competitividade do cluster pelo desenvolvimento de inovação mediante a introdução de tecnologias. Foi possível observar uma atuação direta (i) auxiliando na criação de empresas com propriedade intelectual, desde a designação de CEO até a elaboração do plano de negócios, e suporte para garantir o investimento; além disso, atuam (ii) auxiliando os pesquisadores nos processos de negociação de licenciamentos e nos convênios colaborativos com as empresas, (iii) licenciando tecnologias na indústria e spin-outs. Por fim, também prestam (iv) auxílio aos pesquisadores na realização de registros de patentes, software, marca, direitos autorais e desenho industrial. Com isso pode-se afirmar que uma função macro das entidades de apoio no desenvolvimento de tecnologia e inovação é:

\section{A5. Auxiliar nos processos de proteção e negociação do conhecimento ou tecnologia gerada}

Por fim, as instituições de apoio têm uma atuação ainda mais direcionada e pontual (i) fomentando a inovação e o empreendedorismo, sendo responsáveis pelo contato e gestão de incubadoras 
e parques tecnológicos, e (ii) incubando empresas propriamente ditas gerando spin-offs em que as universidades se tornam acionistas com os acadêmicos fundadores e os investidores.

Em resumo, diante dos exemplos analisados, pode-se dizer que as organizações de apoio atuam sobre a governança supra empresarial, especificamente nos fatores competitivos: estratégias de resultado orientadas para o cluster, e caráter evolucionário por introdução de tecnologias, mediante o exercício das seguintes atividades, desenhadas na Figura 6. Os dados sustentam as afirmativas teóricas encontradas na literatura, sobre a importância das organizações de apoio, agora incrementadas com um rol de categorias que se mostraram mais importantes e que podem auxiliar no direcionamento de pesquisas futuras.

Figura 6 - Atividades próprias das instituições de apoio para competitividade e inovação

Atividades voltadas para desenvolvimento de estratégias conjuntas orientadas para o aumento da competitividade do cluster

Captar e direcionar recursos.

Promover a colaboração entre as empresas por meio da formação de um ambiente integrador e formação de grupos.

Auxiliar o desenvolvimento de competências no cluster.

Exercer representação em nome do cluster junto ao poder público.

Atividades de estímulo ao caráter evolucionário das empresas do cluster

Auxiliar nos processos de proteção e negociação do conhecimento ou tecnologia gerada.

Auxiliar a criação de novos negócios por meio de incubadoras.

Fonte: Elaborado pelos autores.

\section{Considerações finais}

Esta pesquisa originou-se da necessidade de avançar a discussão acerca do papel das organizações de apoio à governança dos clusters no fomento à competitividade destes arranjos, haja vista a escassez de conceitos operacionais e da sua relação com a orientação estratégica e atualização tecnológica dos arranjos. Nesse sentido, analisou-se como as organizações de apoio atuam sobre a governança supra empresarial, especificamente verificando a presença destas organizações e suas respectivas ações de intervenção no desenvolvimento de estratégias conjuntas orientadas para o aumento da competitividade do cluster e estímulo ao caráter evolucionário das empresas aglomeradas.

Conduziu-se uma pesquisa empírica em dois clusters: o Cluster de Ciências da Saúde em Ribeirão Preto, e, o Health Science Cluster em Oxforfdshire na Inglaterra. Ao realizar o estudo em dois casos pertencentes a países com condições sociais e econômicas distintas, foi possível aprofundar a 
análise e explicação do fenômeno pela verificação de similaridades nos clusters em relação aos aspectos pesquisados.

Em ambos os clusters constatou-se a presença de diferentes organizações de apoio às empresas dos clusters, inclusive organizações que apoiam a inovação, estando relacionadas à pesquisa e desenvolvimento de tecnologias. Os discursos indicaram a presença da governança nos clusters, embora alguns a definem como algo ainda pouco demarcado. Essa presença e o grau de desenvolvimento da governança está de acordo com a literatura sobre governança supra empresarial, que a associa a mecanismos relacionais, informal e socialmente construídos, o que significa tempo de maturação e percepção seletiva dos atores, conforme sua posição e imersão na rede (Poppo \& Zenger, 2002; Zacarelli et al., 2008; Arikan \& Schilling, 2011; Bolumole et al, 2015; Bocquet et al., 2016).

Com relação ao fundamento competitivo estratégias de resultado orientadas para o cluster, observou-se que nos dois aglomerados os entrevistados identificaram exemplos de iniciativas estratégicas que se interligam com a governança e geraram ações em prol da competitividade do agrupamento. Sobre o fundamento caráter evolucionário por introdução de tecnologias, também foi possível identificar nos discursos as ações de intervenção na governança com o intuito de estimular a inovação e o desenvolvimento de novas tecnologias nas empresas dos clusters, o que traz ganhos competitivos ao agrupamento.

Com esses achados a pesquisa contribui no aspecto teórico, ao sustentar com evidências a importância das organizações de apoio para a governança do cluster; avançando a compreensão do papel que estas organizações assumem ao desempenhar atividades que fomentam a competitividade dos mesmos, seja no desenvolvimento de ações estratégicas que favoreçam a coletividade, seja na promoção, apoio e disseminação do desenvolvimento tecnológico nas regiões.

Sobre as seis categorias emergentes, houve convergência entre os achados e as afirmativas de Bocquet et al. (2016), sobre captação de recursos; de Provan e Kenis (2008) e Lefebvre (2016) sobre colaboração entre as empresas por meio da formação de um ambiente integrador; de Ingstrup e Damgaard (2013) sobre o desenvolvimento de competências no cluster; de Arikan e Shilling (2011) e Bolumole et al. (2015); sobre representação do cluster junto ao poder público e ações de proteção e negociação do conhecimento ou tecnologia gerada. A convergência dos achados em dois clusters com campos organizacionais distintos abre a possibilidade de se pensar num modelo mais generalista de categorias.

No aspecto prático/gerencial, o artigo contribui ao elencar operacionalmente seis atividades que podem ser fomentadas, planejadas e acompanhadas pelos atores locais (empresários e demais representantes públicos e privados), descritas na Figura 6. Importante salientar que algumas dessas atividades, tais como captar recursos, ou promover a colaboração entre empresas, são usualmente entendidas como de responsabilidade do governo, ou de grandes atores/empresas da rede. $\mathrm{O}$ trabalho mostra que ações gerenciais podem também se dirigir às organizações de apoio. 
$\mathrm{O}$ estudo apresenta algumas limitações, dentre as quais destaca-se o fato de ter priorizado clusters atuantes em um setor específico e de base tecnológica. Dado que clusters não são homogêneos (De Marchi \& Grandinetti, 2014), estudos futuros poderiam minimizar essas limitações desenvolvendo uma pesquisa semelhante em clusters de outros setores que permitiriam evidenciar se o comportamento das instituições de apoio diverge conforme a natureza das atividades dos aglomerados, ou se há tendência de confirmação das categorias que emergiram neste estudo.

\section{Referências}

Angst, M., Hirschi, C. (2017) Network Dynamics in Natural Resource Governance: A Case Study of Swiss Landscape Management. Policy Studies Journal, 45(2): 315-336.

Arikan, A. T. (2009). Interfirm knowledge exchanges and the knowledge creation capability of clusters. Academy of management review, 34(4), 658-676.

Arikan, A. T., \& Schilling, M. A. (2011). Structure and Governance in Industrial Districts: Implications for Competitive Advantage. Journal of Management Studies, 48(4), 772-803.

Bocquet, R., Brion, S., \& Mothe, C. (2016). The Role of Cluster Intermediaries for KIBS' Resources and Innovation. Journal of Small Business Management, 54, 256-277.

Bolumole, Y. A., Closs, D. J., \& Rodammer, F. A. (2015). The Economic Development Role of Regional Logistics Hubs: A Cross-Country Study of Interorganizational Governance Models. Journal of Business Logistics, 36(2), 182-198.

Borgatti, S. et al. (2009) Network analysis in the social sciences. Science, 13(323): 892-895.

Cassanego Junior, P. V., Boaventura, J. M. G., Azevedo, A. C., \& Telles, R. (2019). Governance in business clusters: proposal for an application of an analytical model. Entrepreneurship \& Regional Development, 31(9-10), 984-1010.

Chen, F.; Lin, M.; Wang, T. (2018) Sustainable resource acquisition path: A dynamic model of embedded entrepreneurial network governance under uncertainty. Sustainability, 10(11): 40614086.

Crona, B. \& Parker, J. (2012) Learning in support of governance: Theories, methods, and a framework to assess how bridging organizations contribute to adaptive resource governance. Ecology and Society 17(1):1-25

Cropper, S. et al. (2008) The Oxford Handbook of inter-organizational relations. Oxford: Oxford.

De Marchi, V., \& Grandinetti, R. (2014). Industrial districts and the collapse of the Marshallian model: looking at the Italian experience. Competition \& Change, 18(1), 70-87.

Dyer, J. H., \& Singh, H. (1998). The relational view: Cooperative strategy and sources of interorganizational competitive advantage. Academy of Management Review, 23(4), 660-679.

Eisenhardt, K. M. (1989). Building theories from case study research. Academy of Management Review, 14(4), 532-550. 
Eriksson, P. and Kovalainen, A. (2016), Qualitative Methods in Business Research, Sage, Thousand Oaks, CA.

Fetterman, D. M. (1998). Ethnography. Sage Publications, Inc.

Ferreira, M. A. S. P. V. (2013). A pesquisa e a estruturação do artigo acadêmico em administração. Revista Ibero-Americana de Estratégia, 12(2), 1-11.

FIPASE. Fundação Instituto Polo Avançado da Saúde. (2015). Informações de Ribeirão Preto. Disponível em: http://fipase.org.br/index.php?option=com_content\&view=article \&id=34\&ltemid=28

Gagné, M., Townsend, S. H., Bourgeois, I., \& Hart, R. E. (2010). Technology cluster evaluation and growth factors: Literature review. Research evaluation, 19(2), 82-90.

Gereffi, G., and J. Lee. 2016. Economic and Social Upgrading in Global Value Chains and Industrial Clusters: Why Governance Matters. Journal of Business Ethics 133 (1): 25-38. doi.org/10.1007/s10551-014-2373-7.

Gioia, D. A., Corley, K. G., \& Hamilton, A. L. (2013). Seeking qualitative rigor in inductive research: Notes on the Gioia methodology. Organizational research methods, 16(1), 15-31.

Gorriz-Mifsud, E. et al. (2017) Structural social capital and local-level forest governance: Do they inter-relate? A mushroom permit case in Catalonia. Journal of Environmental Management, 188(3):364-378

Gottschick, M. (2018) Reflexive Capacity in Local Networks for Sustainable Development: Integrating Conflict and Understanding into a Multi-Level Perspective Transition Framework: JEPP Reflexive Governance. Journal of Environmental Policy and Planning, 20(6): 713-734

Grandori, A. (2006) Innovation, Uncertainty and Relational Governance. Industry and Innovation, 13(2), 127-133.

Granovetter, M. (1985). Economic action and social structure: The problem of embeddedness. American journal of sociology, 91(3), 481-510.

Gulati, R., Nohria, N., \& Zaheer, A. (2000). Strategic networks. Strategic management journal, 21(3), 203-215.

Hahl, O.; Kacperczyk, A. \& Davis, J. (2016) Knowledge asymmetry and brokerage: Linking network perception to position in structural holes. Strategic Organization, 14(2): 118-143

Hoppen, N., Rigoni, E. H., Klein, A. Z., \& Ritter, Á. M. (2016). A qualitative research instrument to analyze organizational clusters' competitiveness factors. BASE-Revista de Administração e Contabilidade da Unisinos, 13(1), 2-18.

Humphrey, J., \& Schmitz, H. (2002). How does insertion in global value chains affect upgrading in industrial clusters?. Regional studies, 36(9), 1017-1027.

Ibert, O.; Muller, F. (2015) Network dynamics in constellations of cultural differences: Relational distance in innovation processes in legal services and biotechnology. Research Policy, 44(1):181194. 
Ingstrup, M. B., \& Damgaard, T. (2013). Cluster Facilitation from a Cluster Life Cycle Perspective. European Planning Studies, 21(4), 556-574.

Isis Innovation (2016). About Isis Innovation. Disponível em: http://isis-innovation.com/about/.

Jones, C., Hesterly, W. S., \& Borgatti, S. P. (1997). A general theory of network governance: Exchange conditions and social mechanisms. Academy of management review, 22(4), 911-945.

Kamath, S., Agrawal, J., \& Chase, K. (2012). Explaining geographic cluster success-The GEMS model. American Journal of Economics and Sociology, 71(1), 184-214.

Kathleen, M.; Michael, K. \& Brian, H. (2009) The Etiology of Social Change. Topics in Cognitive Science, 1(4):621-650

Kellogg, W.; Samanta, A. (2018) Network structure and adaptive capacity in watershed governance. Journal of Environmental Planning and Management, 61(1): 25-48

Kim, S. \& Jin, K. (2017) Organizational governance of inter-firm resource combinations: The impact of structural embeddedness and vertical resource relatedness. Journal of Management \& Organization, 23(4): 524-544.

Lefebvre, P. (2016). Cluster Management Organizations as Intermediary Actors of Co-Evolution between Clusters and Entrepreneurial Firms. In C. Boari, T. Elfring, \& X. F. Molina-Morales (Eds.), Entrepreneurship and Cluster Dynamics (1st Edition ed., pp. 248 p.). New York: Routledge.

Li, H., de Zubielqui, G. C., \& O'Connor, A. (2015). Entrepreneurial networking capacity of cluster firms: a social network perspective on how shared resources enhance firm performance. Small Business Economics, 45(3), 523-541.

Lübeck, R. M., Wittmann, M. L., \& da Silva, M. S. (2012). Afinal, quais variáveis caracterizam a existência de cluster arranjos produtivos locais (apls) e dos sistemas locais de produção e inovação (SLPIs)?. Revista Ibero Americana de Estratégia, 11(1), 120-151.

Maccio, L., \& Cristofoli, D. (2017). How to support the endurance of long-term networks: The pivotal role of the network manager. Public Administration, 95(4), 1060-1076.

Miele, V.; Matias, C. (2017) Revealing the hidden structure of dynamic ecological networks. Royal Society open science, 4(6), p.170251-170261

Miles, M. B., \& Huberman, A. M. (1994). Qualitative data analysis: A sourcebook. Beverly Hills: Sage Publications.

Moeliono, M. et al. (2016) Local governance, social networks and REDD+: Lessons from Swidden communities in Vietnam. Human Ecology, 44(4): 435-448

Molina-Morales, F. \& Martínez-Cháfer, L. (2016) Cluster Firms: You'll Never Walk Alone. Regional Studies, 50(5):877-893.

Morosini, P. (2004). Industrial clusters, knowledge integration and performance. World development, 32(2), 305-326.

Mueller, E. F., \& Jungwirth, C. (2016). What drives the effectiveness of industrial clusters? Exploring the impact of contextual, structural and functioning determinants. Entrepreneurship and Regional Development, 28(5-6), 424-447. 
Niesten, E. et al. (2017) Sustainable collaboration: The impact of governance and institutions on sustainable performance. Journal of Cleaner Production, 155(2): 1-6

Ouro Filho, A. M., Leon Olave, M. E., \& De Carvalho Barreto, I. D. (2015). Fatores desarticuladores da cooperação em arranjos produtivos locais: um estudo quantitativo no APL de Confecções de Tobias Barreto/SE. BBR-Brazilian Business Review, 12(5).

Oxfordshire Lep. (2014). Driving Economic Growth Through Innovation. Strategic Economic Plan.

Partelow, S., \& Nelson, K. (2020). Social networks, collective action and the evolution of governance for sustainable tourism on the Gili Islands, Indonesia. Marine Policy, 112.

Patton, M. Q. (1990). Qualitative evaluation and research methods. SAGE Publications, inc.

Paul, F. (2012) Smart business networks: interaction-coordination aspects and risks. Business Process Management Journal, 18(5), p.829-843

Peng, B.; Tu, Y.; Wei, G. (2018) Governance of electronic waste recycling based on social capital embeddedness theory. Journal of Cleaner Production, 187(20): 29-36.

Pereira, C. E. C., Sarturi, G., Boaventura, J. M. G., \& Polo, E. F. (2014). Desenvolvimento de Métricas para Avaliação da Competitividade de Clusters: uma aplicação empírica no setor têxtil. Gestão \& Regionalidade (Online), 30(90), 155-172.

Petry, J. F., Amal, M., \& Floriani, D. E. (2018). Institutional Distance, Regional Clusters and Performance of Foreign Subsidiaries: Evidences from Brazil. BBR. Brazilian Business Review, 15(3), 302-316.

Poppo, L., \& Zenger, T. (2002). Do formal contracts and relational governance function as substitutes or complements?. Strategic management journal, 23(8), 707-725.

Porter, M. E. (1990). The competitive advantage of nations. New York: The Free Press

Porter, M. E. (1998). Clusters and the new economics of competition (Vol. 76, No. 6, pp. 77-90). Boston: Harvard Business Review.

Porter, M. E. (2008). On competition. Harvard Business Press.

Prim, A. L., Amal, M., \& Carvalho, L.. (2016). Regional Cluster, Innovation and Export Performance: An Empirical Study. BAR - Brazilian Administration Review, 13(2), 2-26.

Provan, K. \& Kennis, P. (2008) Modes of networks governance: Structure, management and effectiveness. Journal of Public Administration Research and Theory, 18(2): 229-252.

Roth, A. L., Wegner, D., Valle Antunes Júnior, J. A., \& Domingos Padula, A. (2012). Diferenças e inter-relações dos conceitos de governança e gestão de redes horizontais de empresas: contribuições para o campo de estudos. Revista de Administração-Rausp, 47(1): 112-123.

Sarturi, G., Vargas, C. A. F., Boaventura, J. M. G., \& Santos, S. A. D. (2016). Competitiveness of clusters: A comparative analysis between wine industries in Chile and Brazil. International Journal of Emerging Markets, 11(2), 190-213.

Souza, J. H., Cappa, J., \& Neves, L. C. (2008). Concentração regional da indústria de produtos médicos. São Paulo em Perspectiva (22)1,123-136. 
Thomaz, J. C., Brito, E. P. Z., Marcondes, R. C., \& Ferreira, F. C. M. (2011). Benefícios da aglomeração de firmas: evidências do arranjo produtivo de semijoias de Limeira. Revista de Administração, 46(2), 191-206.

Todeva, E. (2006). Business networks: strategy and structure. London: Routledge.

Usp Inovação. (2016) Educação continuada. Disponível em: http://inovacao.usp.br/educacao/gepit/.

Van Aken, J. E., \& Weggeman, M. P. (2000). Managing learning in informal innovation networks: overcoming the Daphne-dilemma. R\&D Management, 30(2), 139-150.

Visser, E. J., \& Atzema, O. (2008). With or without clusters: Facilitating innovation through a differentiated and combined network approach. European Planning Studies, 16(9), 1169-1188.

Wallenburg, C. \& Schäffler, T. (2014) The interplay of relational governance and formal control in horizontal alliances: A social contract perspective. Journal of Supply Chain Management, 50(2), p.41-58

Wang, L., A. Madhok, and S. X. Li. 2014. "Agglomeration and Clustering over the Industry Life Cycle: Toward a Dynamic Model of Geographic Concentration.” Strategic Management Journal 35 (7): 995-1012. doi.org/10.1002/smj.2141.

Williamson, O. E. (1979). Transaction-cost economics: the governance of contractual relations. The journal of Law and Economics, 22(2), 233-261.

Williamson, O. E. (1996). The mechanisms of governance. Oxford University Press.

Woiceshyn, J., \& Daellenbach, U. (2018). Evaluating inductive vs deductive research in management studies: Implications for authors, editors, and reviewers. Qualitative Research in Organizations and Management: An International Journal, 13(2), 183-195.

Woo, S. E., O'Boyle, E. H., \& Spector, P. E. (2017). Best practices in developing, conducting, and evaluating inductive research. Human Resource Management Review, 2(27), 255-264.

Zaccarelli, S., Telles, R., Siqueira, J., Boaventura, J. and Donaire, D. (2008). Clusters e redes de negócios: uma nova visão para a gestão dos negócios. Atlas.

Zhang, Y., \& Li, H. (2010). Innovation search of new ventures in a technology cluster: the role of ties with service intermediaries. Strategic Management Journal, 31(1), 88-109. 